\title{
Nomogram to Predict Distant Metastasis Probability for Pathological Complete Response Rectal Cancer Patients After Neoadjuvant Chemoradiotherapy
}

\author{
Ting Jiang ${ }^{1, *}$ \\ Shuang Liu',* \\ Xiaojun $\mathrm{Wu}^{2}$,* \\ Xiaoqing $\mathrm{Liu}^{3}$ \\ Weizhan $\mathrm{Li}^{4}$ \\ Shanfei Yang (iD) \\ Peiqiang $\mathrm{Cai}^{5}$ \\ Shaoyan $\mathrm{Xi}^{6}$ \\ Zhifan Zeng (ID) \\ Yuanhong Gao' \\ Gong Chen ${ }^{2}$ \\ Weiwei Xiao'
}

'Department of Radiation Oncology, State Key Laboratory of Oncology in South China, Collaborative Innovation Center for Cancer Medicine, Sun Yat-Sen University Cancer Center, Guangzhou, People's Republic of China; ${ }^{2}$ Department of Colorectal Surgery, Sun Yat-Sen University Cancer Center, Guangzhou, People's Republic of China; ${ }^{3}$ Department of Radiation Oncology, Guangzhou Concord Cancer Center, Guangzhou, People's Republic of China; ${ }^{4}$ Department of Radiation Oncology, Panyu Center Hospital, Guangzhou, People's Republic of China; ${ }^{5}$ Department of Radiology, Sun Yat-Sen University Cancer Center, Guangzhou, People's Republic of China; ${ }^{6}$ Department of Pathology, Sun Yat-Sen University Cancer Center, Guangzhou, People's Republic of China

*These authors contributed equally to this work

Correspondence: Gong Chen; Weiwei

Xiao

Email chengong@sysucc.org.cn;

xiaoww@sysucc.org.cn
Purpose: This study aimed to predict the risks of distant metastasis (DM) of locally advanced rectal cancer (LARC) patients with pathological complete response (pCR) after neoadjuvant chemoradiotherapy (NACRT) and total mesorectal excision (TME), and to find the association between adjuvant chemotherapy (ACT) and their survival outcomes.

Methods and Materials: A total of 242 patients with LARC achieving pCR after NACRT were enrolled in this retrospective study. We developed a nomogram model using logistic regression analyses for predicting risk of DM. The model performance was evaluated by the concordance index and calibration curve. Survival was determined using Kaplan-Meier survival curve.

Results: Age, pre-operative CEA, pre-treatment CEA and distance of tumor to anal verge were identified as significantly associated variables that could be enrolled in the model to predict the risk of DM for pCR patients. The nomogram we created had a bootstrappedconcordance index of $0.731(95 \% \mathrm{CI}=0.627$ to 0.834$)$ and was well calibrated. The high risk group was more likely to develop DM than low risk group (total score) (95\% CI $=1.439$ to 6.493, $P=0.0036$ ). The 1-year, 3-year, and 5-year distant metastasis-free survival (DMFS) for the low and high risk groups (total score $\leq 90$ vs $>90$ ) was $97.8 \%, 94.2 \%, 94.2 \%$ and $91.3 \%, 83.4 \%, 81.8 \%$, respectively $(P=0.0036)$. DM occurred within 1 and 2 years after TME surgery was $33.3 \%$ and $55.6 \%$ for the low risk group, and $47.3 \%$ and $84.2 \%$ for the high risk group. The value of ACT was assessed among the whole cohort, patients with $\mathrm{cT}_{3-4}$, with $\mathrm{cN}_{+}$or with either DM risk group, but no significant difference was observed concerning DMFS whether ACT was given or not (all $P>0.05$ ). Active treatment after DM was more beneficial than palliative treatment $(P<0.001)$.

Conclusion: The nomogram model, including age, pre-operative CEA, pre-treatment CEA and distance to anal verge, predicted the probability of DM among LARC patients achieving pCR after NACRT. The effects of ACT were not seen in different subgroups, while closer clinical follow-up may have greater contribution to pCR patients in the first 2 years, especially for patients with relatively higher risk to develop DM. It is suggested that timely active treatment can bring survival benefit for pCR patients developing DM after NACRT.

Keywords: locally advanced rectal cancer; LARC, pathological complete response; pCR, adjuvant chemotherapy; ACT, distant metastasis; DM

\section{Introduction}

The standard treatment for locally advanced rectal cancer (LARC) is neoadjuvant fluoropyrimidine $(5 \mathrm{Fu})$-based chemoradiotherapy followed by radical resection. More and more clinical trials are trying to investigate how to improve outcomes 
of this patient population. According to a meta-analysis including four large clinical trials, addition of Oxaliplatin decreased distant failure $(\mathrm{DF})(\mathrm{OR}=0.67,95 \% \mathrm{CI}=0.60$ $0.97, P=0.03) .{ }^{1}$ Besides, a promising strategy - total neoadjuvant therapy (TNT) implied that intensive chemotherapy before surgery could reduce $23.7 \%$ in diseaserelated treatment failure in RAPIDO trial; and in PRODIGE 23 it could lead to an absolute 7\% reduction in the risk of DM at 3 years. ${ }^{2}$ The explorations not only improved long-term outcomes but also short-term effects. About $15-30 \%$ of patients achieved pathological complete response (pCR) after neoadjuvant chemoradiotherapy (NACRT) and preserve optimistic prognosis. ${ }^{3,4} \mathrm{pCR}$ is also defined as post-operative stage $\mathrm{T}_{0} \mathrm{~N}_{0}$ which represents that tumor underwent great response to NACRT. We hypothesize that the therapeutic and follow-up strategies to pCR patients after NACRT and total mesorectal excision (TME) should be planned differently according to distinct prognostic factors. $\mathrm{pCR}$ almost eradicated tumor local recurrence possibility and distant metastasis (DM) became the predominant pattern of treatment failure. ${ }^{5}$ Identification of pCR patients at high risk of DM is of great clinical importance. Therefore, we conducted the present study to predict the probability of DM, which might be helpful in counseling follow-up strategies.

Though the role of adjuvant chemotherapy (ACT) in pCR patients is debated, there were also studies demonstrating the positive value of $\mathrm{ACT}^{6-9}$ Since 2017, the National Comprehensive Cancer Network (NCCN) guidelines recommend all patients with clinical stage II/III to accept ACT regardless of the post-operative pathologic staging. ${ }^{10}$ In sharp contrast to NCCN guidelines, the European Society for Medical Oncology (ESMO) guidelines recommend ACT only in yp stage III and "high-risk" yp stage II patients after neoadjuvant chemotherapy or chemoradiotherapy. ${ }^{11}$ Several large studies based on National Cancer Database (NCDB) reported a portion of LARC patients with $\mathrm{pCR}$, especially those with clinical $\mathrm{T}_{3-4}$ staging and positive nodes before treatment, accepting ACT could achieve higher overall survival (OS) rate than those with observation. ${ }^{12,13}$ However, a multi-center retrospective study including 1041 LARC patients with pCR implied that similar OS, local recurrence-free survival (LRFS), distant metastasis-free survival (DMFS) and disease-free survival (DFS) were observed between the ACT group and the non-ACT group. ${ }^{14}$ Obviously, the true value of ACT in pCR patients remains to be determined.
Here our study aimed to predict the probability of DM of pCR patients and to propose the necessity of close follow-up mode for some of $\mathrm{pCR}$ patients. We developed a nomogram adopting pre-operative factors to predict high risks to DM probability among patients with pCR after NACRT and TME surgery. We also estimated the association of ACT with the prognosis in LARC patients achieving pCR after NACRT and sought to reveal the connection of $\mathrm{ACT}$ and $\mathrm{pCR}$ patients with different DM probability.

\section{Methods and Materials}

\section{Patients Characteristics}

In all, 1011 patients diagnosed with LARC from March 2009 to July 2017, in a single tertiary Cancer Center were enrolled in our study. Criteria for selecting pCR patients were as follows: 1) Clinical stage II-III rectal cancer at diagnosis; 2) Pelvic imaging (CT and/or MRI) before and after NACRT; 3) Without other concurrent malignant tumors, or ever; 4) Without severe complications; 5) Neoadjuvant chemotherapy based on Capecitabine \pm Oxaliplatin with long-course radiation; 6) TRG 1 based on Mandard's five-tier grading system; and 7) Without adjuvant radiation therapy. Together we identified 242 pCR patients $(23.9 \%)$. The baseline characteristics and treatment details were listed in Table 1.

\section{Neoadjuvant Chemoradiation Therapy}

Radiotherapy was administered with 3D-CRT (9.9\%), IMRT/VMAT $(89.3 \%)$ or tomotherapy techniques $(0.8 \%)$, delivering a total dose of 48-55 Gy to macroscope tumor (containing primary lesion and enlarged lymph nodes) and 45 Gy to lymphatic drainage regions. Radiation was performed as one fraction per day, 5 days per week, and in total 5-5.5 weeks.

Neoadjuvant chemotherapy was mainly administered in two modes (94.2\%): 2 cycles of Capecitabine or 4 cycles of CAPEOX containing 1, 2, 1 cycles before, during and after irradiation, respectively.

\section{Total Mesorectal Excision}

All patients received TME with 5-12 weeks interval after radiation, including Dixon in 180 patients $(74.4 \%)$ and Miles in 62 patients (25.6\%). Among 136 patients (56.2\%) with colostomy, 74 patients received preventive colostomies, of whom 68 patients underwent the closure. Laparoscopic surgery was given in 107 patients (44.2\%). Ascites on exploration of the abdominal cavity were seen 
Table I Patients Characteristics and Survival Analysis

\begin{tabular}{|c|c|c|c|c|c|c|}
\hline Characteristics & & Total (\%) 247 (100) & OS Rates (\%) & PI & DFS Rates (\%) & $\mathbf{P 2}$ \\
\hline Tumor size $(\mathrm{cm})$ & - & $6.2 \pm 2.1$ & & - & & - \\
\hline Radiation dose (Gy) & - & $49.4 \pm 2.0$ & & - & & - \\
\hline Gender & $\begin{array}{l}\text { Male } \\
\text { Female }\end{array}$ & $\begin{array}{l}166(67.2) \\
81(32.8)\end{array}$ & $\begin{array}{l}95.2 \\
95.1\end{array}$ & 0.997 & $\begin{array}{l}91.0 \\
87.7\end{array}$ & 0.457 \\
\hline Age (year) & $\begin{array}{l}\leq 38 \\
>38\end{array}$ & $\begin{array}{l}32(13.0) \\
215(87.0)\end{array}$ & $\begin{array}{l}93.8 \\
95.3\end{array}$ & 0.743 & $\begin{array}{l}78.1 \\
91.6\end{array}$ & 0.018 \\
\hline Family history & $\begin{array}{l}\text { No } \\
\text { Yes }\end{array}$ & $\begin{array}{l}192(77.7) \\
45(24.1)\end{array}$ & $\begin{array}{l}95.4 \\
94.5\end{array}$ & 0.806 & $\begin{array}{l}88.5 \\
94.5\end{array}$ & 0.205 \\
\hline Distance to anal $(\mathrm{cm})$ & $\begin{array}{l}\leq 5 \\
>5 \text { and } \leq 7 \\
>7\end{array}$ & $\begin{array}{l}I 56(63.2) \\
52(2 \mid . I) \\
39(I 5.8)\end{array}$ & $\begin{array}{l}94.2 \\
96.2 \\
97.4\end{array}$ & 0.691 & $\begin{array}{l}91.7 \\
90.4 \\
82.1\end{array}$ & 0.219 \\
\hline Pre-treatment CEA (ng/mL) & $\begin{array}{l}\leq 3 \\
>3 \text { and } \leq 9.5 \\
>9.5\end{array}$ & $\begin{array}{l}112(45.3) \\
90(36.4) \\
45(18.2)\end{array}$ & $\begin{array}{l}94.6 \\
96.7 \\
93.3\end{array}$ & 0.637 & $\begin{array}{l}92.9 \\
90.0 \\
82.2\end{array}$ & 0.083 \\
\hline MRF & $\begin{array}{l}\text { Negative } \\
\text { Positive }\end{array}$ & $\begin{array}{l}\text { I } 28(5 \mathrm{I} .8) \\
119(48.2)\end{array}$ & $\begin{array}{l}96.9 \\
93.3\end{array}$ & 0.147 & $\begin{array}{l}90.6 \\
89.1\end{array}$ & 0.516 \\
\hline EMVI & $\begin{array}{l}\text { Negative } \\
\text { Positive }\end{array}$ & $\begin{array}{l}167(67.6) \\
80(32.4)\end{array}$ & $\begin{array}{l}95.2 \\
95.0\end{array}$ & $0.84 I$ & $\begin{array}{l}90.4 \\
88.8\end{array}$ & 0.537 \\
\hline Clinical T stage & $\begin{array}{l}\mathrm{T} 2 \\
\mathrm{~T} 3 \\
\mathrm{~T} 4\end{array}$ & $\begin{array}{l}\text { I3 (5.3) } \\
172(69.6) \\
62(25.1)\end{array}$ & $\begin{array}{l}100.0 \\
95.3 \\
93.5\end{array}$ & 0.558 & $\begin{array}{l}100 \\
89.0 \\
90.3\end{array}$ & 0.428 \\
\hline Clinical $\mathrm{N}$ stage & $\begin{array}{l}\text { N0 } \\
\text { NI } \\
\text { N2 }\end{array}$ & $\begin{array}{l}67(27.1) \\
77(3 \mid .2) \\
103(4 \mid .7)\end{array}$ & $\begin{array}{l}94.0 \\
97.4 \\
94.2\end{array}$ & 0.543 & $\begin{array}{l}91.0 \\
85.7 \\
92.2\end{array}$ & 0.328 \\
\hline Risk category & $\begin{array}{l}\text { Good } \\
\text { Intermediate } \\
\text { Bad } \\
\text { Ugly }\end{array}$ & $\begin{array}{l}29(11.7) \\
45(18.2) \\
19(7.7) \\
154(62.3)\end{array}$ & $\begin{array}{l}96.6 \\
95.6 \\
100.0 \\
94.2\end{array}$ & 0.676 & $\begin{array}{l}89.7 \\
93.3 \\
89.5 \\
89.0\end{array}$ & 0.813 \\
\hline NACRT & $\begin{array}{l}\text { Xeloda*2 } \\
\text { Xelox*2 } \\
\text { Xelox*3 } \\
\text { Xelox*4 } \\
\text { Others }\end{array}$ & $\begin{array}{l}46(18.6) \\
34(13.8) \\
42(17.0) \\
110(44.5) \\
15(6.1)\end{array}$ & $\begin{array}{l}100.0 \\
97.1 \\
88.1 \\
97.3 \\
80.0\end{array}$ & 0.004 & $\begin{array}{l}93.5 \\
88.2 \\
83.3 \\
93.6 \\
73.3\end{array}$ & 0.042 \\
\hline Radiation Tech & $\begin{array}{l}\text { 3D-CRT } \\
\text { VMAT/IMRT } \\
\text { ToMo }\end{array}$ & $\begin{array}{l}25(10.1) \\
220(89.1) \\
2(0.8)\end{array}$ & $\begin{array}{l}92.0 \\
95.5 \\
100\end{array}$ & 0.801 & $\begin{array}{l}88.0 \\
90.0 \\
100\end{array}$ & 0.908 \\
\hline Pre-operative CEA (ng/mL) & $\begin{array}{l}\leq 2 \\
>2\end{array}$ & $\begin{array}{l}104(42.1) \\
143(57.9)\end{array}$ & $\begin{array}{l}95.2 \\
95.1\end{array}$ & 0.970 & $\begin{array}{l}93.3 \\
87.4\end{array}$ & 0.129 \\
\hline Surgery & $\begin{array}{l}\text { Dixon } \\
\text { Miles } \\
\text { Hartmann } \\
\text { TEM }\end{array}$ & $\begin{array}{l}\text { I } 80(72.9) \\
64(25.9) \\
2(0.8) \\
I(0.4)\end{array}$ & $\begin{array}{l}96.7 \\
90.6 \\
100.0 \\
100.0\end{array}$ & 0.265 & $\begin{array}{l}90.6 \\
87.5 \\
100 \\
100\end{array}$ & 0.839 \\
\hline
\end{tabular}


Table I (Continued).

\begin{tabular}{|l|l|l|l|l|l|l|}
\hline Characteristics & & Total (\%) 247 (100) & OS Rates (\%) & PI & DFS Rates (\%) & P2 \\
\hline Surgical approach & Open & $139(56.3)$ & 95.0 & 0.998 & 88.5 \\
& Laparoscopic & $\begin{array}{l}108(43.7) \\
\text { Colostomy }\end{array}$ & No & $10743.3)$ & 95.4 & 91.7 \\
& Yes & $140(56.7)$ & 97.2 & 9.563 \\
\hline Complication for surgery & No & $223(90.3)$ & 95.1 & 89.7 & 90.0 \\
& Yes & $24(9.7)$ & 95.8 & 0.823 & 89.7 & 91.7 \\
\hline ACT & No & $60(24.3)$ & 93.3 & 0.409 & 91.7 & 0.741 \\
& Yes & $187(75.7)$ & 95.7 & & 89.3 \\
\hline
\end{tabular}

Abbreviations: OS, overall survival; DMFS, distant metastasis-free survival; CEA, carcinoembryonic antigen; MRF, mesorectal fascia; EMVI, extramural vascular invasion; NACRT, neoadjuvant chemoradiotherapy; Radiation Tech, radiation technology; ACT, adjuvant chemotherapy.

in 24 patients $(9.9 \%)$. There were 22 cases $(9.1 \%)$ with more than $200 \mathrm{~mL}$ intra-operative bleeding. Twenty-one cases were assessed as difficult operations by surgeons and 9 patients underwent multiple organ resection. The resected specimens were examined by pathologists specialized in gastrointestinal cancers. pCR was assessed as no remaining tumor cells.

Post-operative complications occurred in 24 patients $(9.8 \%)$, which contained anastomotic leakage in 8 patients, post-operative bowel obstruction in 5 , rectovaginal fistula in 2, wound dehiscence in 6 and other complications in 3 patients.

\section{Adjuvant Chemotherapy}

After radical resection, 183 patients $(75.6 \%)$ were treated with Capecitabine-based ACT, whereas 59 patients (24.4\%) underwent observation. In ACT group, 68 patients accepted Capecitabine treatment following CAPEOX; 74 patients accepted different cycles CAPEOX and 41 patients accepted single agent Capecitabine as their ACT.

\section{Follow-Up}

The first follow-up visit was performed approximately one month after surgery. And then patients were followed up every 3-6 months until death or the cut-off date (August 31, 2020). We searched the medical records, took telephone calls and checked population registration system to update the follow-up. OS was calculated from the initial radical surgery to the last follow-up or death. DMFS was defined as the duration from the initial radical resection to the last follow-up or the first detection of DM. We identified oligo-metastases as cancer involving 1-3 lesions per organ with a maximum tumor diameter less than $7 \mathrm{~cm}$ through expert radiologists. ${ }^{15}$

\section{Statistical Analysis}

All statistical tests were performed in $\mathrm{R}$ project (Version 3.6.0), X-tile (Version 3.6.1) and IBM SPSS statistics (Version 23.0). The least absolute shrinkage and selection operator (LASSO) method in Cox regression was used to select the most useful predictive features from the primary data. ${ }^{16}$ The optimal value of cut-off was determined by X-tile. ${ }^{17}$ The nomogram was generated using the rms package within $\mathrm{R}$ project. The nomogram performance was measured using concordance index (C-index) and assessed by calibration curves as previously described. ${ }^{18}$ Furthermore, all possible model combinations of selected predictive features were constructed. After comparisons, the final model with the highest $\mathrm{C}$-index was reported. Model validation was performed by bootstrapping using 1000 resamples.

We constructed Kaplan-Meier survival curves to estimate the OS and DMFS of the whole cohort and different subgroups $\left(\mathrm{cT}_{3-4}\right.$ group, $\mathrm{cN}_{+}$group and high/low risk group in the nomogram model). Statistical significance was defined as $\mathrm{P}<0.05$. Survival curves were depicted using GraphPad Prism 8 software.

\section{Results}

\section{Treatment Outcomes}

Until follow-up, only 1 patient had local recurrence. There were 28 patients developing DM. Until the last follow-up, 11 patients $(4.5 \%)$ passed away due to DM. The median OS for the whole cohort was 53.0 months (10.6-112.8 
Table 2 Distant Metastasis Events in 28 Patients

\begin{tabular}{|c|c|c|c|}
\hline \multirow[t]{2}{*}{ Endpoint } & & \multicolumn{2}{|c|}{ Multivariate Analysis } \\
\hline & & HR (95\% Cl) & $P$ \\
\hline OS & $\begin{array}{l}\text { MRF (postive vs. } \\
\text { negative) } \\
\text { NACRT } \\
\text { Others } \\
\text { Xeloda*2 } \\
\text { Xelox*2 } \\
\text { Xelox*3 } \\
\text { Xelox*4 }\end{array}$ & $\begin{array}{l}1.082(0.477-2.454) \\
\overline{-} \\
0.219(0.048-1.004) \\
0.321(0.076-1.359) \\
0.216(0.150-1.778) \\
0.199(0.057-0.693)\end{array}$ & $\begin{array}{l}0.851 \\
0.094 \\
0.051 \\
0.123 \\
0.295 \\
0.011\end{array}$ \\
\hline DFS & $\begin{array}{l}\text { Age }(>38 \text { vs. } \leq 38) \\
\text { Pre-treatment CEA } \\
(\mathrm{ng} / \mathrm{mL}) \\
\leq 3 \\
>3 \text { and } \leq 9.5 \\
>9.5 \\
\text { Pre-operative CEA } \\
(\mathrm{ng} / \mathrm{mL}) \\
\text { NACRT } \\
\text { Others } \\
\text { Xeloda*2 } \\
\text { Xelox*2 } \\
\text { Xelox*3 } \\
\text { Xelox*4 }\end{array}$ & $\begin{array}{l}0.331 \\
\\
\overline{1.319}(0.132-0.829) \\
2.627(0.472-3.687) \\
1.642(0.620-4.345) \\
\\
\\
0.303 \\
0.420(0.065-1.404) \\
0.541 \\
0.246(0.153-1.917) \\
(0.069-0.870)\end{array}$ & $\begin{array}{l}0.018 \\
\\
0.177 \\
0.598 \\
0.077 \\
0.318 \\
\\
0.244 \\
0.127 \\
0.228 \\
0.342 \\
0.030\end{array}$ \\
\hline
\end{tabular}

Abbreviations: MRF, mesorectal fascia; NACRT, neoadjuvant chemoradiotherapy; OS, overall survival; DFS, disease-free survival.

months), The 1-year, 3-year, 5-year OS was 99.6\%, $95.7 \%, 94.7 \%$, respectively.

The median DMFS of these 28 patients was 13.4 months (0.93-62.57 months) (Table 2). The 1-year, 3-year, 5-year DMFS was 95.0\%, 89.6\%, 88.9\%, respectively. Metastatic organs included 1 with bone, 1 with liver, 10 with lung and 16 with multiple metastases to lymph nodes, as we shown in Table 2.
Oligo-metastases occurred in 12 of 28 patients $(42.9 \%)$, among whom 10 patients received ablation or local resection; 1 patient accepted both local resection and chemotherapy. The rest 1 with vertebral metastasis died before their planned surgery. Poly-metastases occurred in 16 patients $(57.1 \%)$. Nine cases had systemic chemotherapy in our Cancer Center, whereas 7 patients underwent palliative treatment at local hospitals. Patients with oligo-metastases had significant better survival outcomes than patients with poly-metastases $(P=0.002$, Figure 1A). $91.7 \%$ and $56.3 \%$ of patients with oligometastases and poly-metastases were confirmed within 2 years after TME surgery, respectively (Figure 1B). Those 20 patients $(71.4 \%)$ who received local treatment \pm systemic chemotherapy, systemic chemotherapy and palliative treatment, had significant different survival outcomes (Figure $1 \mathrm{C}, P=0.002$ ).

\section{Nomogram Development and Internal Validation}

For the development of nomogram, we incorporated 72 clinical features as prognostic features (Supplementary Table 1). All these parameters were reduced to the most useful 4 potential predictive features for DMFS, with nonzero coefficients in the LASSO cox regression model (Supplementary Figure 1). Age, pre-operative CEA, pretreatment CEA and distance to anal verge were independent prognostic factors (Figure 2A). The predictive accuracy for DMFS as measured by C-index was 0.731 (95\% CI $=0.627$ to 0.834 ) in the internal validation. The calibration plots for the probability of 1-, 3-, and 5-year DMFS also exhibited good consistency between the predicted DMFS and the actual DMFS (Figure 2B-D).
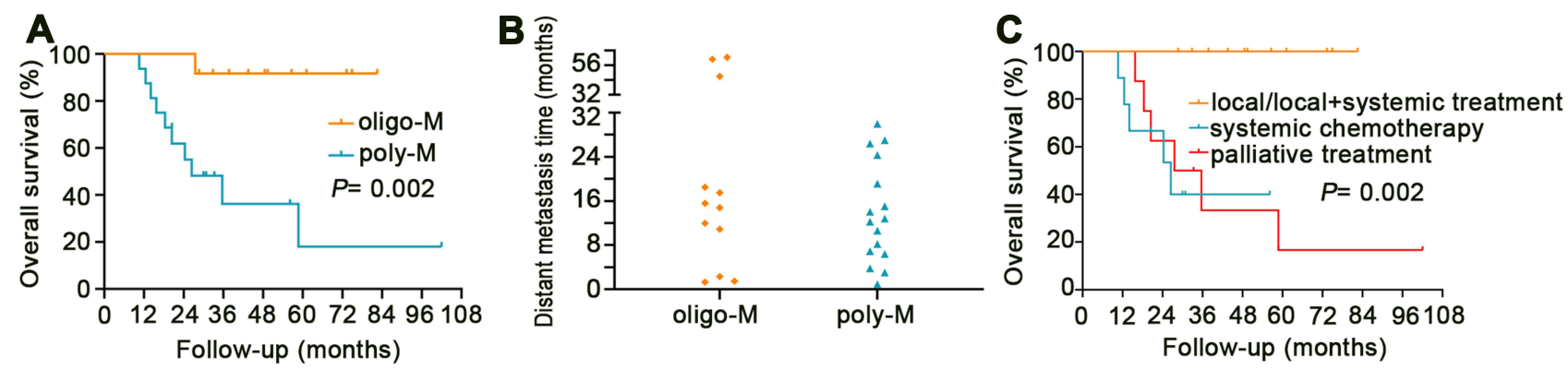

Figure I The Kaplan-Meier curves for PCR patients with metastasis in the follow-up. (A) The overall survival curves for different distant metastasis patterns. (B) Time distribution of different distant metastasis after TME. (C) The overall survival curves for PCR patients developing metastasis during the follow-up stratified by different treatment strategies.

Abbreviations: Oligo-M, oligo-metastasis; poly-M, poly-metastasis; TME, total mesorectal excision. 
A

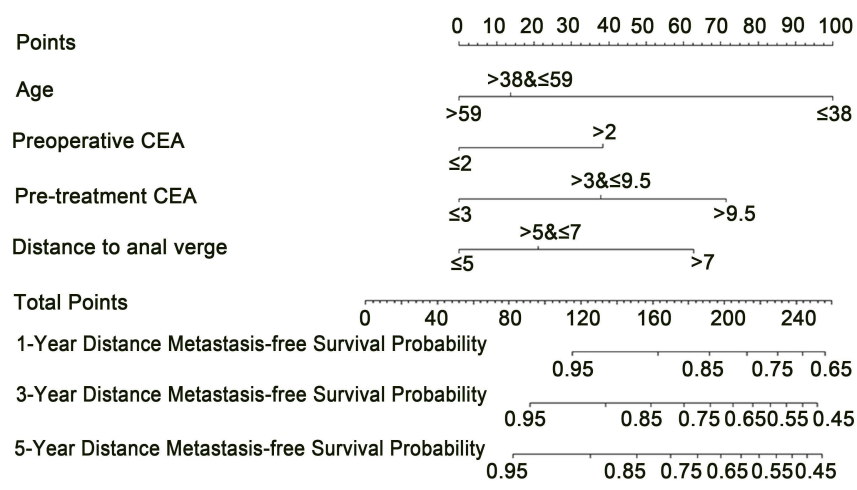

C

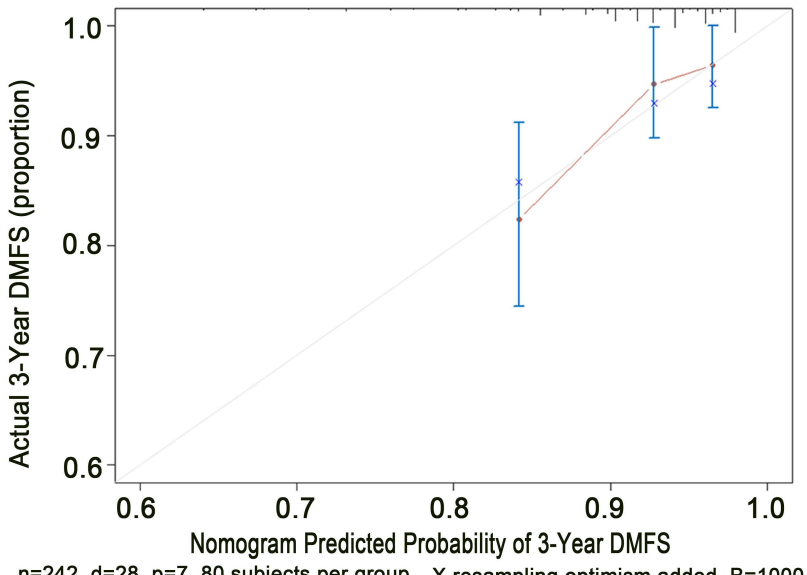

$n=242, d=28, p=7,80$ subjects per group $X$ resampling optimism added, $B=1000$ Gray: ideal
B

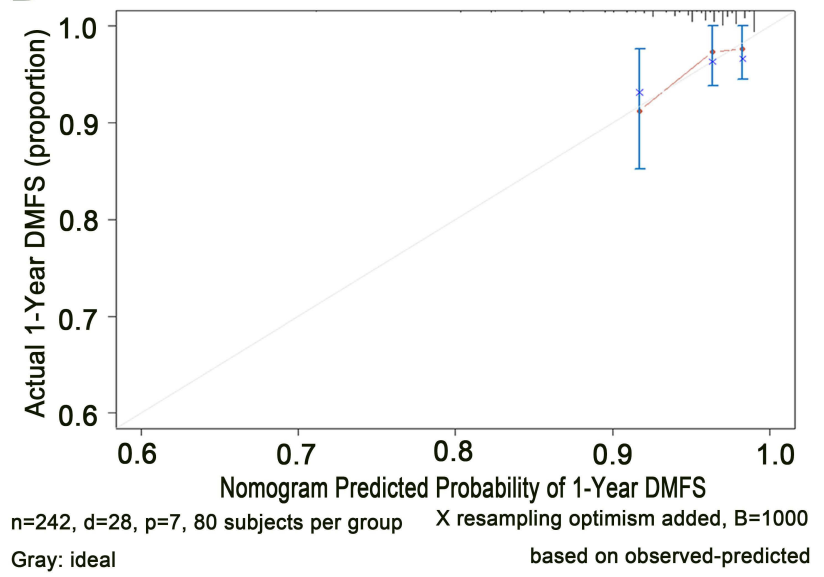

D

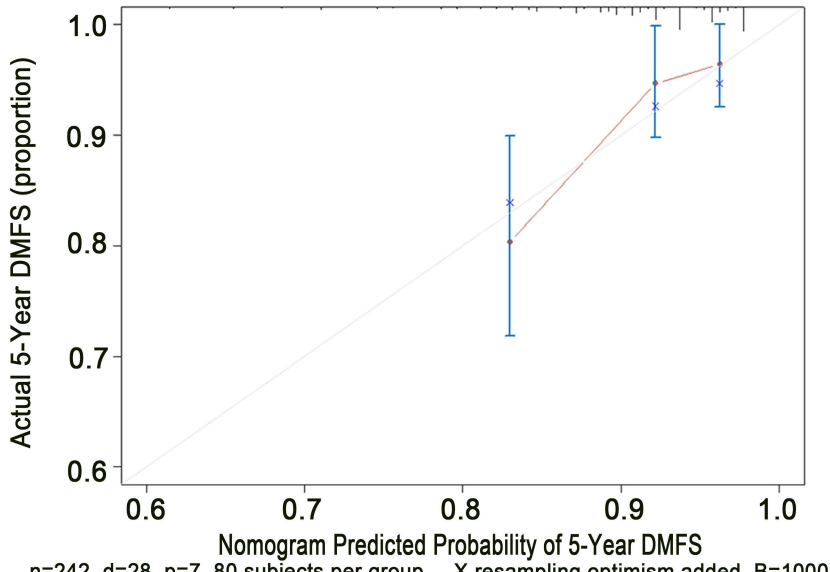

$n=242, d=28, p=7,80$ subjects per group $X$ resampling optimism added, $B=1000$ Gray: ideal based on observed-predicted

Figure 2 Construction and validation of nomogram in rectal cancer patients achieving pCR after neoadjuvant chemoradiotherapy. (A) Nomogram for predicting I-, 3-, and 5-year distant metastasis-free survival probability for patients. (B-D) Calibration curves of nomograms in terms of agreement between predicted and observed I-, 3-, and 5 -year outcomes.

Each subtype within these variables was assigned a score on the point scale. By calculating the total score, we classified 103 pCR patients with total score $>90$ into high risk group (42.6\%) and 139 patients with total score $\leq$ 90 into low risk group (57.4\%). The 1-year, 3-year, and 5-year DMFS for the low and high risk groups was $97.8 \%$, $94.2 \%, 94.2 \%$, and $91.3 \%, 83.4 \%, 81.8 \%$, respectively ( $\mathrm{P}$ $=0.0036$, Figure 3A). However, OS rates did not show obviously difference between high and low risk group $(P=$ 0.684 , Figure 3B).

As shown in Table 2, there were 9 patients $(6.5 \%)$ who developed DM in the low risk group. Among them 2 patients had oligo-metastases and received local lesions resection. Seven patients had poly-metastases, 5 of them received systemic chemotherapy while the other 2 received palliative treatment. In the high risk group, 19 patients (18.4\%) occurred DM. Among them 10 patients had oligometastases, 8 received local resection or ablation. One patient received systemic chemotherapy following local resection. One patient with bone metastasis did not accept any treatment and died before planned surgery. Nine of 19 patients had poly-metastases. Four patients undertook systemic chemotherapy. And the remaining 5 cases with poly-metastases did not have any chemotherapy possibly due to financial burden or poor physical condition.

DM occurred within 1 and 2 years after TME surgery was $33.3 \%$ and $55.6 \%$ for the low risk group, and $47.3 \%$ and $84.2 \%$ for the high risk group (Figure 3C, Table 2).

\section{Value of Adjuvant Chemotherapy}

Compared with non-ACT patients, patients with the receipt of ACT did not perform a better DMFS $(P=$ 

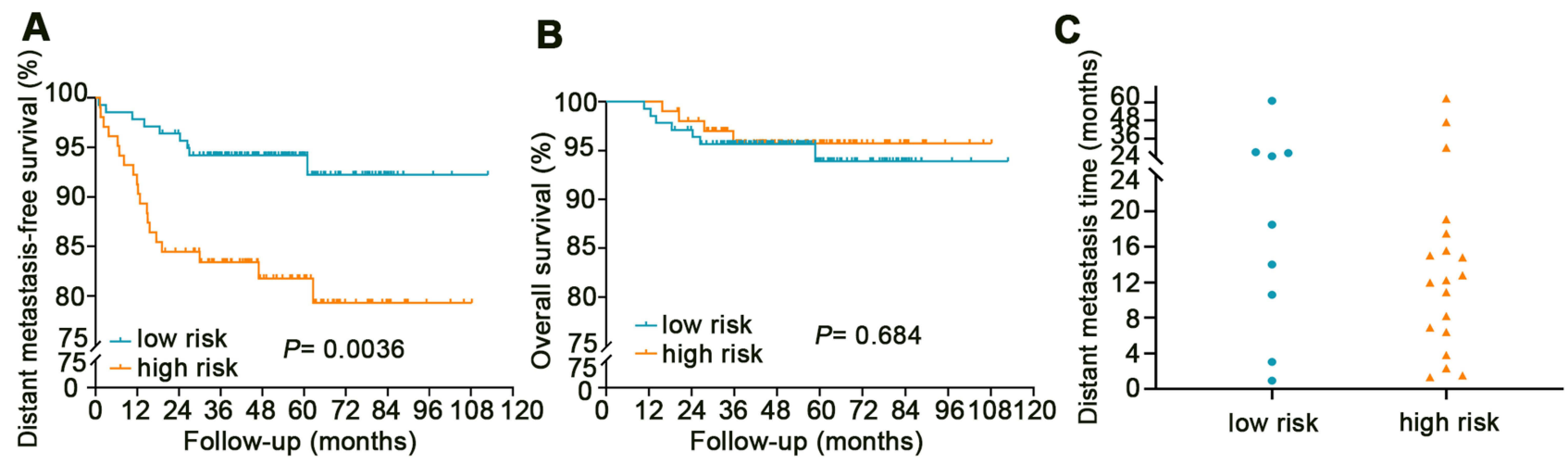

Figure 3 The KaplanMeier survival curves for PCR patients. (A) Distant metastasis-free survival curves for high and low risk groups according to the nomogram model. (B) Overall survival curves for high and low risk groups defined in nomogram model. (C) The distribution of distant metastasis events after TME in low and high risk groups. Abbreviation: TME, total mesorectal excision.

0.962 ) in the overall population (Figure 4A). In the subgroup analysis, ACT was associated with no significant improvement in DMFS neither in $\mathrm{cT}_{3-4}$ subgroup $(P=$ 0.990 , Figure 4B), nor in $\mathrm{cN}_{+}$subgroup $(P=0.845$ for DMFS, Figure $4 C$ ). The value of ACT was also assessed in high and low risk groups divided using our nomogram model, as it was shown in Figure 4D-E, DMFS was similar between ACT and non-ACT groups in both high and low risk subgroups (all $P>0.05$ ).

The role of ACT was also assessed when using OS as an endpoint. However, it was similar between ACT and non-ACT groups (Supplementary Figure 2A-E).

\section{Discussion}

Over the past decades, chemoradiotherapy performed before radical surgery has largely decreased local recurrence rates and improved survival outcomes amongst rectal cancer patients. However, high metastatic potential keeps a big threaten to these patients. Numerous randomized trials have been conducted to investigate the efficacy of NACRT in rectal cancer patients. As a novel therapeutic approach, TNT, showed excellent effect on local control and DM as well as tumor down-staging. ${ }^{19}$ The population of pCR was enlarged gradually. Regardless of their favorable prognosis, DM is still a serious problem, which warrants more attention.

Few studies tried to analyzed the DM risk in LARC patients with $\mathrm{pCR}$, who had great response to NACRT. Development of DM is the major cause of mortality among colorectal cancer (CRC) patients. ${ }^{20,21}$ Our result was in agreement with SUN's study which indicated that DM remained a major concern also in pCR patients following NACRT and TME. ${ }^{7}$ Age, pre-operative CEA, pre- treatment CEA and distance to anal verge were performed as independent prognostic factors to construct our nomogram model. Despite pCR patients had better survival outcome with high 5-year DMFS rate up to $88.9 \%$, our nomogram still had enough power to predict the distinct probability of DM $(\mathrm{C}$-index $=0.731)$, in which total score $>90$ had significantly higher risks to metastasis.

In our nomogram, age showed the highest relative weight and young patients along with poorer prognosis. Generally, patients under age 40 are classified as young. The incidence of CRC in this group is increasing. Our study was consistent with the findings of previous studies that younger patients ( $\leq 38$ years old, our data) with $\mathrm{CRC}$ showed higher degree of malignancy and higher probability of DM than the older patients. ${ }^{22}$ Although age is not universally considered as an independent factor at present, young patients have been reported to have a more aggressive biological and worst prognosis generally attributed to the discovery of a more advanced disease, as stages III and IV predominate among young patients who are not regularly recommended colonoscope examination for CRC..$^{23,24}$ Recently a team created a first-generation metastasis map to reveal organ-specific patterns of metastasis through marking human cells before injecting to nude mice. ${ }^{25}$ In the analysis process, they found that decreased metastatic potential was accompanied by increased patient age.

CEA, a high molecular-weight glycoprotein belonging to the immunoglobulin superfamily of molecules, is secreted to the cell surface by tumor cells and identified as a prominent metastatic adhesion molecular in CRC cells. ${ }^{26}$ It is commonly accepted as an independent prognostic factor of CRC. In our nomogram, both pretreatment and pre-operative CEA is associated with the 


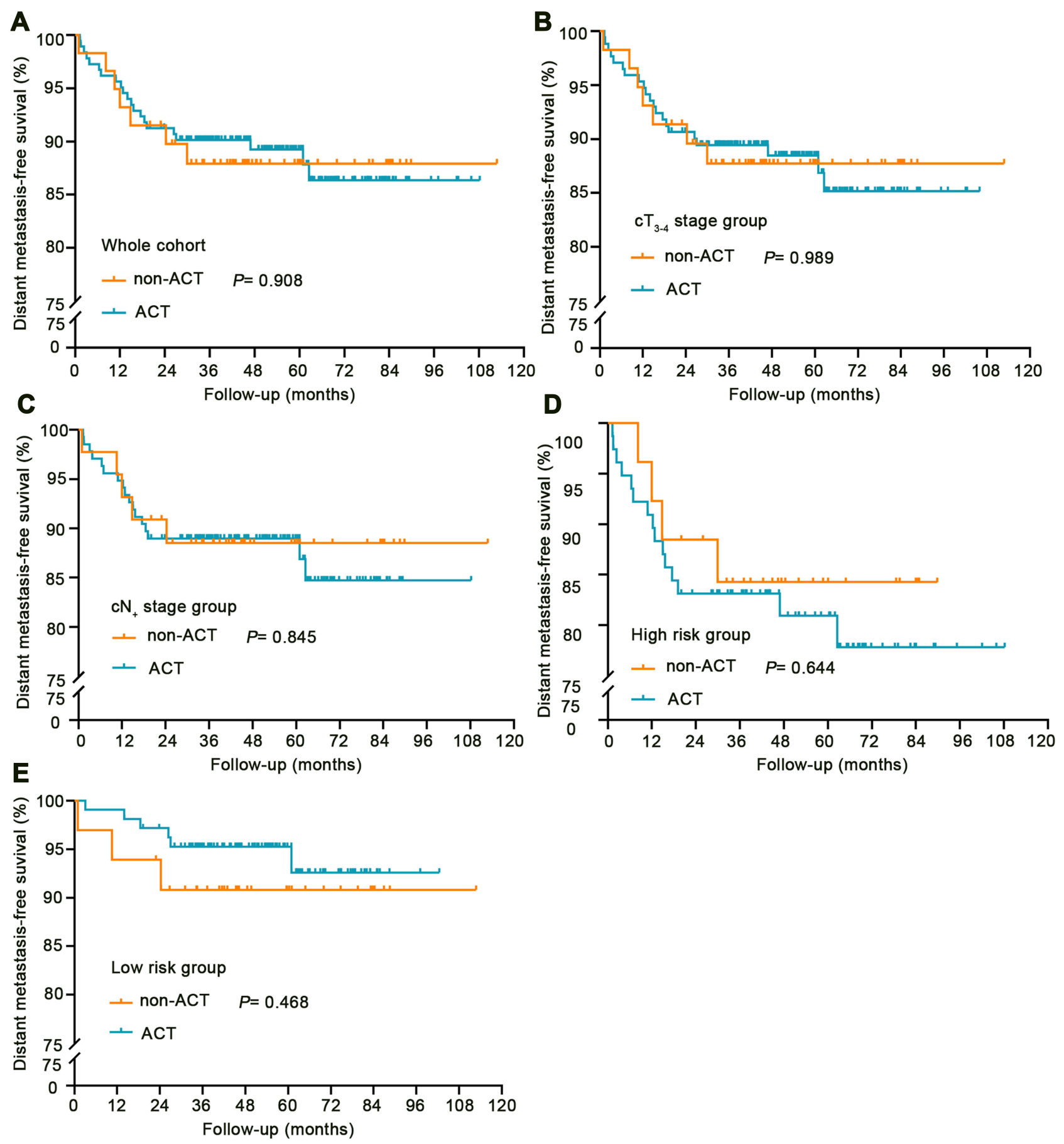

Figure 4 The Kaplan-Meier survival curves of distant metastasis-free survival stratified by adjuvant chemotherapy for pCR patients. The whole cohort (A) and different subgroups (B-E), including $\mathrm{cT}_{3-4}$ stage group $(\mathbf{B}), \mathrm{cN}_{+}$stage group $(\mathbf{C})$, high risk group (D) and low risk group (E).

DMFS probability of $\mathrm{pCR}$ patients. Kim et al reported that the reduction ratio of pre-treatment and pre-operative CEA may be an independent prognostic factor for DFS in rectal cancer patients. ${ }^{27}$ Nevertheless, their conclusion had a prerequisite condition-pre-treatment CEA $>6 \mathrm{ng} / \mathrm{mL}$. Though the two CEA levels have certain relationship in medicine, it is appropriate to use the two features in the model, which avoids omitting detailed information. In our nomogram, high serum CEA concentration $>2 \mathrm{ng} / \mathrm{mL}$ before operation and $>9.5 \mathrm{ng} / \mathrm{mL}$ before treatment is more likely to occur DM.

In our study, we found that the distance to anal verge was positively correlated with DM rates. The nearer the tumor was located from anal verge, the lower DM risks 
patients would have. In a retrospective research including 562 patients with nonmetastatic rectal cancer with NACRT and TME, Das et al demonstrated that the distance from the anal verge $>5 \mathrm{~cm}$ predicted lower tumor downstaging $(\mathrm{HR}=0.46,95 \%$ CI $0.25-0.83, P=0.010) .{ }^{28}$ Recently, Deng's team developed a nomogram to predict $\mathrm{pCR}$ and good response in LARC. They concluded that the greater distance of the tumor from the anal verge was independent predictors of low rates of $\mathrm{pCR}(\mathrm{OR}=0.82,95 \%$ CI 0.69 $0.96, P=0.019)$ and downstaging $(\mathrm{OR}=0.56,95 \% \mathrm{CI}$ $0.33-0.95, P=0.032){ }^{29}$ The macroscopic tumor with shorter distance to anal verge might represent that the lesions are sensitive to chemoradiation and patients might have fewer micrometastasis after treatment. In the long run, we could infer these patients with lower distance to anal verge may have lower risk of DM. However, the unique biological characteristics of $\mathrm{pCR}$ patients should be unfolded through further basic experiments to validate our hypothesis. Also, it is necessary to confirm our model in prospective clinical trials.

We noticed that most pCR patients developed DM in 2 years. If patients with metastatic lesions could receive timely treatment, including local and systemic management, their progressed lesions could have opportunity to be controlled well and they are more likely to have longterm survival compared with patients in palliative treatment group. ESMO guidelines recommend a minimum of two CTs of the chest, abdomen and pelvis in the first 3 years; NCCN guidelines recommend that patients with stage II-III need perform these imaging examinations every 3-6 month for a total of 5 years. According to our study, pCR patients should accept CTs of the chest, abdomen and pelvis every 3 months for the first 2 years after surgery. The most appropriate follow-up mode is worth further exploring.

Though the model could predict DM of pCR patients, the OS rates between high and low risk groups had no apparent differences, which may due to great prognosis and few deaths of pCR patients (Supplementary Figure 2D-E). We suppose a much larger pCR cohort with longer time follow-up is needed to explore whether patients in low risk group have better OS rates or not.

The efficacy of ACT discussed in previous studies is still controversial in pCR patients who has favorable prognosis which requires large-scale samples to show differences. Analysis based on NCDB suggested some patients who achieved pCR after NACRT could get benefit from $\mathrm{ACT},{ }^{6,8}$ especially in those who were more possible to had poor prognosis, like patients with $\mathrm{cT}_{3-4} \mathrm{~N}_{+}(\mathrm{HR}=0.47$, 95\% CI, 0.25-0.91, $P=0.005) .{ }^{13}$ But several prospective studies indicated that ACT is unnecessary for NACRTinduced pCR LARC patients. ${ }^{14,30,31}$ Agreeing with their study, patients with or without ACT had comparable OS rates and DMFS rates in our data. In stratified groups, patients with $\mathrm{cT}_{3-4}, \mathrm{cN}_{+}$and high risks to $\mathrm{DM}$ had no obvious benefit from ACT.

Patients included in this cohort were all from one hospital, whose imaging examination before treatment was elaborately reviewed by doctors in terms of the unified criterions. Combining MRF, EMVI and other information, we classified all patients to different ESMO risk groups. ${ }^{32}$ We also recorded chemotherapy and surgery in details. After assessing multiple factors related with DM, our nomogram containing four factors is plausible and easy to use to predict DM probability of pCR patients. In terms of associated cost of systemic ACT, our study suggests it would be more valuable to adopt close follow-up and timely management, especially in patients with high risk of DM defined by the nomogram model.

To our knowledge, we performed a retrospective study about pCR patients from one cancer center with detailed clinical information and long follow-up. So, it has certain inherent limitations. Although the current study has a relatively large sample size for this special kind of patients, an independent internal cohort and external cohort will further confirm the reliability of predictive model.

\section{Conclusions}

In conclusion, we constructed a nomogram model including age, pre-operative CEA, pre-treatment CEA and distance to anal verge to predict the probability of DMFS among pCR rectal patients after NACRT and TME surgery. ACT is not recommended for pCR patients. Closer clinical follow-up strategy is recommended to pCR patients so that DM can be timely controlled, especially in the first 2 years, for those patients with high DM risk defined by the nomogram model.

\section{Abbreviations}

DM, distant metastasis; LARC, locally advanced rectal cancer; pCR, pathological complete response; NACRT, neoadjuvant chemoradiotherapy; TME, total mesorectal excision; OS, overall survival; LRFS, local recurrencefree survival; DMFS, distant metastasis-free survival; DFS, disease-free survival; NCDB, National Cancer Database; NCCN, National Comprehensive Cancer 
Network; ESMO, European Society for Medical Oncology; CEA, carcinoembryonic antigen; MRF, mesorectal fascia; EMVI, extramural vascular invasion; Radiation Tech, radiation technology.

\section{Data Sharing Statement}

The data sets used and analyzed during the current study are available from the corresponding author (Weiwei Xiao) on reasonable request.

\section{Code Availability}

The authors declare that all data and R scripts supporting the findings of this study are available from the corresponding author (Weiwei Xiao) upon reasonable request.

\section{Ethical Approval}

The present study was performed in accordance with the ethical standards as presented in the 1964 Declaration of Helsinki and its later amendments or comparable ethical standards and was approved by the Ethics Committee of Sun Yat-sen University Cancer Center with a waiver of informed consent because this research was retrospective and did not involve accessing any patient identification data.

\section{Acknowledgments}

We thank the medical staff and patients for their contribution to this study. Ting Jiang, Shuang Liu and Xiaojun Wu contributed equally to this work and shared the first authorship.

\section{Author Contributions}

All authors made a significant contribution to the work reported, whether that is in the conception, study design, execution, acquisition of data, analysis and interpretation, or in all these areas; took part in drafting, revising or critically reviewing the article; gave final approval of the version to be published; have agreed on the journal to which the article has been submitted; and agree to be accountable for all aspects of the work.

\section{Funding}

This work was supported by the 5010 Clinical Research Foundation of Sun Yat-sen University (Grant number 5010-2018-04).

\section{Disclosure}

The authors declare that they have no conflicts of interest.

\section{References}

1. De Felice F, Benevento I, Magnante AL, et al. Clinical benefit of adding oxaliplatin to standard neoadjuvant chemoradiotherapy in locally advanced rectal cancer: a meta-analysis: oxaliplatin in neoadjuvant treatment for rectal cancer. BMC Cancer. 2017;17(1):325. doi:10.1186/s12885-017-3323-4

2. Giunta EF, Bregni G, Pretta A, et al. Total neoadjuvant therapy for rectal cancer: making sense of the results from the RAPIDO and PRODIGE 23 trials. Cancer Treat Rev. 2021;96:102177. doi:10.1016/j.ctrv.2021.102177

3. Maas M, Nelemans PJ, Valentini V, et al. Long-term outcome in patients with a pathological complete response after chemoradiation for rectal cancer: a pooled analysis of individual patient data. Lancet Oncol. 2010;11(9):835-844. doi:10.1016/S1470-2045(10)70172-8

4. Zorcolo L, Rosman AS, Restivo A, et al. Complete pathologic response after combined modality treatment for rectal cancer and long-term survival: a meta-analysis. Ann Surg Oncol. 2012;19 (9):2822-2832. doi:10.1245/s10434-011-2209-y

5. Fan WH, Xiao J, An X, et al. Patterns of recurrence in patients achieving pathologic complete response after neoadjuvant chemoradiotherapy for rectal cancer. J Cancer Res Clin Oncol. 2017;143 (8):1461-1467. doi:10.1007/s00432-017-2383-9

6. Shahab D, Gabriel E, Attwood K, et al. Adjuvant chemotherapy is associated with improved overall survival in locally advanced rectal cancer after achievement of a pathologic complete response to chemoradiation. Clin Colorectal Cancer. 2017;16(4):300-307. doi:10.1016/j.clcc.2017.03.005

7. Sun Y, Wu X, Zhang Y, et al. Pathological complete response may underestimate distant metastasis in locally advanced rectal cancer following neoadjuvant chemoradiotherapy and radical surgery: incidence, metastatic pattern, and risk factors. Eur J Surg Oncol. 2019;45 (7):1225-1231. doi:10.1016/j.ejso.2019.03.005

8. Ma B, Ren Y, Chen Y, et al. Is adjuvant chemotherapy necessary for locally advanced rectal cancer patients with pathological complete response after neoadjuvant chemoradiotherapy and radical surgery? A systematic review and meta-analysis. Int J Colorectal Dis. 2019;34 (1):113-121. doi:10.1007/s00384-018-3181-9

9. Morris MC, Winer LK, Lee TC, Shah SA, Rafferty JF, Paquette IM. Omission of adjuvant chemotherapy in rectal cancer patients with pathologic complete response: a national analysis. $J$ Gastrointest Surg. 2020;1-9.

10. Benson AB, Venook AP, Al-Hawary MM, et al. Rectal cancer, Version 2.2018, NCCN clinical practice guidelines in oncology. J Natl Compr Canc Netw. 2018;16(7):874-901. doi:10.6004/jnccn.2018.0061

11. Glynne-Jones R, Wyrwicz L, Tiret E, et al. Rectal cancer: ESMO clinical practice guidelines for diagnosis, treatment and follow-up. Ann Oncol. 2018;29(Suppl 4):iv263. doi:10.1093/annonc/mdy161

12. Dossa F, Acuna SA, Rickles AS, et al. Association between adjuvant chemotherapy and overall survival in patients with rectal cancer and pathological complete response after neoadjuvant chemotherapy and resection. JAMA oncol. 2018;4(7):930-937. doi:10.1001/jamaoncol.20 17.5597

13. Polanco PM, Mokdad AA, Zhu H, Choti MA, Huerta S. Association of adjuvant chemotherapy with overall survival in patients with rectal cancer and pathologic complete response following neoadjuvant chemotherapy and resection. JAMA oncol. 2018;4(7):938-943. doi:10. 1001/jamaoncol.2018.0231

14. He F, Ju HQ, Ding Y, et al. Association between adjuvant chemotherapy and survival in patients with rectal cancer and pathological complete response after neoadjuvant chemoradiotherapy and resection. Br J Cancer. 2020;123(8):1244-1252. doi:10.1038/s414 16-020-0989-1 
15. Hellman S, Weichselbaum RR. Oligometastases. J Clin Oncol. 1995;13(1):8-10. doi:10.1200/JCO.1995.13.1.8

16. Sauerbrei W, Royston P, Binder H. Selection of important variables and determination of functional form for continuous predictors in multivariable model building. Stat Med. 2007;26(30):5512-5528. doi: $10.1002 / \operatorname{sim} .3148$

17. Camp RL, Dolled-Filhart M, Rimm DL. X-tile: a new bio-informatics tool for biomarker assessment and outcome-based cut-poin optimization. Clin Cancer Res. 2004;10(21):7252-7259. doi:10.11 58/1078-0432.CCR-04-0713

18. Harrell FE Jr., Lee KL, Mark DB. Multivariable prognostic models: issues in developing models, evaluating assumptions and adequacy, and measuring and reducing errors. Stat Med. 1996;15(4):361-387. doi:10.1002/(SICI)1097-0258(19960229)15:4<361::AID-SIM168>3. $0 . \mathrm{CO} ; 2-4$

19. Liu S, Jiang T, Xiao L, et al. Total neoadjuvant therapy (TNT) versus standard neoadjuvant chemoradiotherapy for locally advanced rectal cancer: a systematic review and meta-analysis. oncologist. 2021. doi:10.1002/onco.13824

20. Habr-Gama A, São Julião GP, Gama-Rodrigues J, et al. Baseline $\mathrm{T}$ classification predicts early tumor regrowth after nonoperative management in distal rectal cancer after extended neoadjuvant chemoradiation and initial complete clinical response. Dis Colon Rectum. 2017;60(6):586-594. doi:10.1097/DCR.0000000000000830

21. Sauer R, Liersch T, Merkel S, et al. Preoperative versus postoperative chemoradiotherapy for locally advanced rectal cancer: results of the German CAO/ARO/AIO-94 randomized Phase III trial after a median follow-up of 11 years. J Clin Oncol. 2012;30(16):1926-1933. doi:10.1200/JCO.2011.40.1836

22. Siegel RL, Miller KD, Goding sauer A, et al. Colorectal cancer statistics, 2020. CA Cancer J Clin. 2020;70(3):145-164. doi: $10.3322 /$ caac. 21601

23. Chou CL, Chang SC, Lin TC, et al. Differences in clinicopathological characteristics of colorectal cancer between younger and elderly patients: an analysis of 322 patients from a single institution. $\mathrm{Am}$ J Surg. 2011;202(5):574-582. doi:10.1016/j.amjsurg.2010.10.014
24. Du CZ, Zhang JS, Li M, et al. [Comparison of pathologic and clinical characteristics of young and old patients with advanced rectal cancer after neoadjuvant radiotherapy]. Zhonghua Wai Ke Za Zhi. 2010;48 (21):1616-1620. [Indonesian]

25. Jin X, Demere Z, Nair K, et al. A metastasis map of human cancer cell lines. Nature. 2020;588(7837):331-336. doi:10.1038/s41586020-2969-2

26. Bird NC, Mangnall D, Majeed AW. Biology of colorectal liver metastases: a review. J Surg Oncol. 2006;94(1):68-80. doi:10.1002/ jso. 20558

27. Kim CW, Yu CS, Yang SS, et al. Clinical significance of pre- to post-chemoradiotherapy s-CEA reduction ratio in rectal cancer patients treated with preoperative chemoradiotherapy and curative resection. Ann Surg Oncol. 2011;18(12):3271-3277. doi:10.1245/ s10434-011-1740-1

28. Das P, Skibber JM, Rodriguez-Bigas MA, et al. Predictors of tumor response and downstaging in patients who receive preoperative chemoradiation for rectal cancer. Cancer. 2007;109(9):1750-1755. doi:10.1002/cncr.22625

29. Zhang JW, Cai Y, Xie XY, et al. Nomogram for predicting pathological complete response and tumor downstaging in patients with locally advanced rectal cancer on the basis of a randomized clinical trial. Gastroenterol Rep. 2020;8(3):234-241. doi:10.1093/gastro/ goz073

30. Hu X, Li YQ, Ma XJ, Zhang L, Cai SJ, Peng JJ. Adjuvant chemotherapy for rectal cancer with complete pathological response (pCR) may not be necessary: a pooled analysis of 5491 patients. Cancer Cell Int. 2019;19(1):127. doi:10.1186/s12935-019-0851-9

31. Gamaleldin M, Church JM, Stocchi L, Kalady M, Liska D, Gorgun E. Is routine use of adjuvant chemotherapy for rectal cancer with complete pathological response justified? Am J Surg. 2017;213 (3):478-483. doi:10.1016/j.amjsurg.2016.11.028

32. Glynne-Jones R, Wyrwicz L, Tiret E, et al. Rectal cancer: ESMO clinical practice guidelines for diagnosis, treatment and follow-up. Ann Oncol. 2017;28(suppl_4):iv22-iv40. doi:10.1093/annonc/mdx224

\section{Publish your work in this journal}

Cancer Management and Research is an international, peer-reviewed open access journal focusing on cancer research and the optimal use of preventative and integrated treatment interventions to achieve improved outcomes, enhanced survival and quality of life for the cancer patient.
The manuscript management system is completely online and includes a very quick and fair peer-review system, which is all easy to use. Visit http://www.dovepress.com/testimonials.php to read real quotes from published authors. 